\title{
Compuestos de interés funcional del nanche (Byrsonima crassifolia (L.) H.B.K.)
}

Compounds of functional interest of nanche (Byrsonima crassifolia (L.) H.B.K.)

\section{Cindy Sacnithe Agredano-de la Garza ${ }^{1}$ (D) , Graciela Guadalupe López-Guzmán ${ }^{2^{*}}$ (D) , Rosendo Balois-Morales $^{2}$ (D) Andrés Eloy León-Fernández ${ }^{3}$ (ID) , Pedro Ulises Bautista-Rosales ${ }^{2}$ (iD) , Yolotzin Apatzingán Palomino-Hermosillo ${ }^{2}$ (D) , José Orlando Jiménez-Zurita ${ }^{2}$ (D) , Edgar Iván Jiménez-Ruiz ${ }^{2}$ (D) , Porfirio Juárez-López ${ }^{4}$}

${ }^{1}$ Posgrado en Ciencias Biológico-Agropecuarias, Universidad Autónoma de Nayarit (UAN), Ciudad de la Cultura s/n, 63000, Tepic, Nayarit, México. ${ }^{2}$ Secretaría de Investigación y Posgrado (UAN).

${ }^{3}$ Instituto Tecnológico del Sur de Nayarit, Carretera Crucero Ahuacatlán-Jala Km 4.5, 63880, Jala, Nayarit, México.

${ }^{4}$ Facultad de Ciencias Agropecuarias, Universidad Autónoma del Estado de Morelos, Av. Universidad 1001, Col. Chamilpa, 62209, Cuernavaca, Morelos, México.

*Autor para correspondencia: lguzman2303@hotmail.com

Fecha de recepción:

14 de abril de 2020

Fecha de aceptación:

15 de enero de 2021

Disponible en línea:

12 de agosto de 2021

Este es un artículo en acceso abierto que se distribuye de acuerdo a los términos de la licencia Creative Commons.

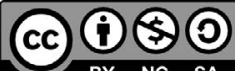

Reconocimiento-

NoComercia-

CompartirIgual 4.0

Internacional

\section{RESUMEN}

El fruto de nanche (Byrsonima crassifolia (L.) H.B.K.) es un producto de consumo en fresco que aporta un alto contenido de compuestos bioactivos con actividad antioxidante, vitamina $\mathrm{C}$ y fibra dietética. Además, se ha reportado que el fruto, hojas y semillas poseen propiedades antioxidantes, antimicrobianas, antiinflamatorias, antiproliferativas, antidepresivas y antidiabéticas. El objetivo principal de esta investigación fue la recopilación de información de los últimos diez años sobre las propiedades fisicoquímicas, nutricionales y compuestos bioactivos funcionales para la salud (polifenoles, flavonoides, vitaminas, antocianinas, fibra) del fruto de nanche.

\section{PALABRAS ClAVE}

Antioxidantes, carotenoides, fenoles, fibra, fitoquímicos.

\section{ABSTRACT}

Nance fruit (Byrsonima crassifolia (L.) H.B.K.) is a product for fresh consumption that provides a high content of bioactive compounds with antioxidant activity, vitamin $\mathrm{C}$, and dietary fiber. In addition, it has been reported that the fruit, leaves, and seeds have antioxidant, antimicrobial, anti-inflammatory, antiproliferative, anti-depressive, and antidiabetic properties. The main objective of this research was the collection of information from the last 10 years on the Nance fruit physicochemical, nutritional and functional bioactive compounds that are beneficial to health (polyphenols, flavonoids, vitamins, anthocyanins, fiber).

\section{KEYWORDS}

Antioxidants, carotenoids, phenols, fiber, phytochemicals 


\section{INTRODUCCIÓN}

El nanche Byrsonima crassifolia (L.) H.B.K. recibe diferentes nombres, dependiendo del país o región donde se desarrolla: Nance, nancite (América Central), peralejo (Cuba), nanche, nanchi, changunga (México), indano (Perú), muricí (Brasil), golden spoon o golden cherry (EUA). Byrsonima crassifolia es una especie nativa de México y América Central, perteneciente a la familia Malpighiacea (Argueta y Mata 2009; conABIo 2019). En México, se encuentran establecidas en producción comercial aproximadamente 1,574 ha, en Guerrero, Nayarit y Michoacán, con rendimiento promedio de 5.38 t.ha $^{-1}$ (SIAP 2018).

El nanche es muy apreciado como complemento alimenticio por su gran aporte de vitaminas y minerales (tiamina, riboflavina y niacina); también es una excelente fuente de fibra dietética (Gordon et al. 2011; Rufino et al. 2010). Adicionalmente, proporciona diferentes compuestos bioactivos antioxidantes, como compuestos fenólicos, carotenoides (luteína y zeaxantina) y vitamina C (Almeida et al. 2011; Mariutti et al. 2013). Los principales compuestos bioactivos contenidos en el fruto de nanche son el ácido gálico y quercetina (Pires et al. 2019; Rodríguez et al. 2016). También contiene cantidades traza de catequina, epicatequina, rutina y kaempferol (Mariutti et al. 2013). Diversas investigaciones han demostrado el efecto antioxidante de las hojas, fruto y semillas de nanche, a las cuales se les atribuyen propiedades antinflamatorias, antiproliferativas y antihiperglicémicas (Carlos et al. 2017; Herrera-Ruiz et al. 2011; Pérez-Gutiérrez et al. 2010).

El fruto de nanche en madurez de consumo presenta diferentes coloraciones, entre las cuales se destaca el amarillo; asimismo, posee una pulpa suave y carnosa, fuerte aroma característico y sabor variable según su contenido de solidos solubles totales y $\mathrm{pH}$ del fruto (Rivas-Castro et al. 2019; Medina-Torres et al. 2015). Es consumido principalmente en estado fresco y en forma de jugos, jaleas, helados y licores (Caballero et al. 2012; Moreno-Martínez et al. 2016). Aunque el fruto tiene diversos beneficios, su comercialización ha sido restringida a los mercados locales y máximo regionales, debido al bajo volumen de producción agrícola y a que su fruto es altamente perecedero (Pereira y Dos Santos 2015; Moreno-Martínez et al. 2016).
Debido a la creciente demanda de alimentos que contengan antioxidantes naturales y con potencial nutricional, el fruto de nanche es considerado como materia prima factible para la aplicación y desarrollo de productos con propiedades funcionales. Por lo anterior, el objetivo de la presente investigación fue recopilar información científica y conocimiento sobre las características fisicoquímicas, nutricionales y funcionales de este frutal.

\section{Materiales y Métodos}

Se revisó literatura publicada entre 2009 y 2020, utilizando diversas bases de datos (ScienceDirect, Redalyc, SciELO, PubMed, Scopus y Elsiever); los principales temas que se definieron fueron: características fisicoquímicas, nutricionales y funcionales del nanche.

\section{Propiedades y componentes nutricionales del nanche}

El consumidor, por lo general, selecciona los frutos de nanche por sus atributos externos; sin embargo, al momento de ser consumidos deben satisfacer otras exigencias, dadas por la combinación de propiedades intrínsecas, tales como el contenido de sólidos solubles totales, firmeza, color, acidez y $\mathrm{pH}$ del jugo, considerados parámetros de calidad para el consumo y la industria (Medina-Torres et al. 2015). Respecto a sus propiedades físicas y químicas, el sabor depende principalmente de los sólidos solubles y de la acidez del fruto, debido al equilibrio que existe entre azúcares y ácidos orgánicos (Medina-Torres et al. 2015). Los sólidos solubles totales ( ${ }^{\circ}$ Brix) encontrados para pulpa de nanche van de 1.5 a 17.32 por ciento en selecciones analizadas de El Cerrado, Brasil, mientras que, en selecciones analizadas en México, los valores oscilan entre 4 y 15.99 por ciento (Cuadro 1). Otro parámetro de calidad que influye en el sabor del fruto de nanche es la acidez titulable; en frutos analizados en México (0.47-2.6\%), se reportan valores bajos en comparación con los analizados en Brasil (0.17-5.30\%); el pH de frutos de El Cerrado, Brasil, tiene valores de 3.20 a 4.74 por ciento, similares a los reportados para frutos de México (3.4-4.6\%). 
Cuadro 1. Propiedades fisicoquímicas de frutos de nanche (Byrsonima crassifolia (L.) H.B.K.) analizado en diferentes regiones de Brasil y México.

\begin{tabular}{|c|c|c|c|c|c|}
\hline Origen & $\begin{array}{c}\text { SS } \\
\left({ }^{\circ} \text { Brix }\right)\end{array}$ & $\begin{array}{c}\text { AT (g de AA } \\
\left.100 \mathrm{~g}^{-1}\right)\end{array}$ & $\mathrm{pH}$ & 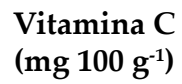 & Referencia \\
\hline \multicolumn{6}{|l|}{ Brasil } \\
\hline Alagoas & 17.32 & 2.44 & 3.35 & 42.24 & Dos Santos et al. (2018) \\
\hline Selva Amazoníca & 5.93 & 0.94 & 3.44 & 3.53 & Pires et al. (2017) \\
\hline Barra do Garças & 12.06 & 0.17 & 4.74 & 92.59 & Morzelle et al. (2015) \\
\hline Palmas & 11.1 & 1.05 & 3.20 & - & Monteiro et al. (2015) \\
\hline Tocantis & 5.20 & 5.30 & 3.64 & 17.45 & Abadio et al. (2012) \\
\hline Selva Amazónica & 1.5 & 1 & 3.7 & - & Canuto et al. (2010) \\
\hline \multicolumn{6}{|l|}{ México } \\
\hline Nayarit & 15.99 & 1.25 & 3.47 & - & Medina-Torres et al. (2015) \\
\hline Yucatán & 8.95 & 0.47 & - & 116.67 & Moo-Huchin et al. (2014) \\
\hline Guanajuato & 14 & 2.6 & 4.62 & 40 & López et al. (2014) \\
\hline
\end{tabular}

El peso del fruto varía de 2.07 a $7.02 \mathrm{~g}$, con forma cilíndrica, alargada o ligeramente alargada (Dos Santos et al. 2018; Hamacek et al. 2014; Medina-Torres et al. 2015; Morzelle et al. 2015; Rivas-Castro et al. 2019). El peso de la semilla oscila entre 0.55 y $1.05 \mathrm{~g}$ (Dos Santos et al. 2018; Hamacek et al. 2014). El rendimiento de la parte comestible del fruto corresponde a cáscara y pulpa, la cual representa entre 76 y 79.04 por ciento, mientras que la semilla es 21.9 por ciento del peso total del fruto (Morzelle et al. 2015; Rivas-Castro et al. 2019).

Diversos autores han reportado que el nanche se caracteriza por presentar un alto contenido de vitaminas y minerales (ácido cítrico, tiamina, riboflavina, niacina) (Moo-Huchin et al. 2014; Morzelle et al. 2015); particularmente, la pulpa es una fuente rica de ácido ascórbico (vitamina C) y carotenoides, que, aunados a sus compuestos fenólicos, hacen sinergia específica en la capacidad antioxidante total presente en este fruto (Almeida et al. 2011). Rufino et al. (2010) afirman que el contenido de Vitamina C varía entre 90-150 mg 100 $\mathrm{g}^{-1}$ de fruto fresco, al igual que Morzelle et al. (2015), quienes reportaron $92.59 \mathrm{mg}$ de vitamina $C$ en frutos provenientes de El Cerrado, Brasil; estos valores superan casi cuatro veces al contenido de vitamina C en naranja (62.50 mg $100 \mathrm{~g}^{-1}$ ) (Rufino et al. 2010).

La vitamina $C$ en cultivares comerciales de nanche (amarillo, verde y rojo) provenientes de Yucatán,

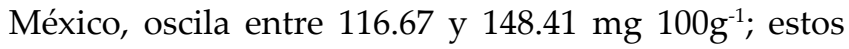
valores son superiores a los reportados en frutos como zapote, zapote mamey, manzana verde y pitahaya
(21.43-37.3 mg $100 \mathrm{~g}^{-1}$ ) (Moo-Huchin et al. 2014). La variabilidad existente en el contenido de vitamina $C$ está determinada con el grado de madurez de los frutos, pues, durante la maduración, los ácidos orgánicos son utilizados para la producción de energía durante el proceso respiratorio (Pimentel et al. 2010; Da Silva et al. 2016). Muchas especies nativas proporcionan frutos que tienen características sensoriales únicas y altas concentraciones de nutrientes; de acuerdo con Rufino et al. (2010), el consumo de frutas tropicales está aumentando en los mercados nacionales e internacionales, debido al creciente reconocimiento del valor nutricional y nutraceútico.

El análisis nutricional del fruto de nanche muestra elevado contenido de humedad en la pulpa (70.9-88.22\%) (Cuadro 2), la cual, junto con la fragilidad de su cáscara, hace que los frutos sean altamente susceptibles al deterioro enzimático y microbiano, lo que dificulta su conservación (Hamacek et al. 2014). Asimismo, el fruto posee un alto contenido de carbohidratos, que van de 7.0 a 21.0 por ciento, lo cual es benéfico para su procesamiento, si se considera que los consumidores prefieren frutos más dulces (Morzelle et al. 2015).

El contenido de proteínas en la pulpa se comporta de manera homogénea $(0.8-1.9 \%)$ para las muestras originarias de Brasil y México. Para la cáscara se reporta 1.89 por ciento, mientras que para la semilla 8.8 por ciento (Araújo et al. 2018; Carlos et al. 2017). Los lípidos en la cáscara $(8.27 \%)$ son mayores a los 
Cuadro 2. Contenido de los componentes nutricionales en frutos de nanche (Byrsonima crassifolia (L.) H.B.K.) por cada $100 \mathrm{~g}$.

\begin{tabular}{|c|c|c|c|c|c|c|c|}
\hline Origen & Humedad & Carbohidratos & Fibra dietética & Proteínas & Lípidos & Cenizas & Referencia \\
\hline \multicolumn{8}{|l|}{ Brasil } \\
\hline Pará & 5.46 & 21.60 & - & 0.55 & 10.02 & 2.02 & $\begin{array}{l}\text { Pires et al. } \\
\text { (2019) }\end{array}$ \\
\hline Amazonia Brasileira & 88.22 & 9.18 & - & 0.86 & 1.45 & 0.30 & $\begin{array}{l}\text { Pires et al. } \\
\quad(2017)\end{array}$ \\
\hline Barra do Garças & 70.9 & 18.85 & 4.98 & 1.94 & 2.31 & 1.02 & $\begin{array}{l}\text { Morzelle et al. } \\
\text { (2015) }\end{array}$ \\
\hline Palmas & 79.46 & 15.23 & 2.02 & 0.82 & 1.78 & 0.69 & $\begin{array}{c}\text { Monteiro et al. } \\
\text { (2015) }\end{array}$ \\
\hline Minas Gerais & 71.58 & 7.47 & 13.58 & 1.26 & 5.13 & 0.93 & $\begin{array}{c}\text { Hamacek et al. } \\
\text { (2014) }\end{array}$ \\
\hline Pará & 76.5 & 15.1 & 3.7 & 1 & 3.4 & 0.7 & $\begin{array}{l}\text { Siguemoto } \\
\text { (2013) }\end{array}$ \\
\hline Tocantins & 78.58 & 18.04 & - & 1.89 & 8.7 & 0.52 & $\begin{array}{l}\text { Abadio et al. } \\
\text { (2012) }\end{array}$ \\
\hline \multicolumn{8}{|l|}{ Estados Unidos } \\
\hline Beltsville & 80.64 & 16.97 & 7.5 & 0.66 & 1.16 & - & USDA (2018) \\
\hline
\end{tabular}

encontrados en pulpa (1.45-3.4\%) (Carlos et al. 2017; Monteiro et al. 2015; Morzelle et al. 2015; Pires et al. 2017); sin embargo, Pires et al. (2019) reportaron 10.02 por ciento (pulpa + cáscara). En el perfil lipídico de la pulpa se presentan aproximadamente 65 por ciento de ácidos grasos monoinsaturados, el oleico como el más común, seguido del palmítico, aunque también se ha detectado el esteárico, así como ácidos poliinsaturados como el linoléico y docosahexaenoico (Pires et al. 2017 Siguemoto 2013). En el caso de la semilla, se han reportado valores del contenido de lípidos de 15.0 a $33.0 \mathrm{~g}$, donde se identifican como principales ácidos grasos al palmítico, oléico y linoléico, los cuales son responsables de 95 por ciento del total de ácidos grasos (Araújo et al. 2018; Costa et al. 2017; Rodríguez et al. 2016).

El nanche también es una importante fuente de fibra dietética, particularmente soluble en pulpa (pectinas, almidones) e insoluble en cáscaras (ligninas y hemicelulosa) (Jiménez et al. 2012). El Departamento de Agricultura de los Estados Unidos (USDA), en 2018, reportó un contenido de 7.5 g de fibra dietética en nanche congelado; por otra parte, Hamacek et al.
(2014) reportaron $13.58 \mathrm{~g}$ de fibra dietética, mientras

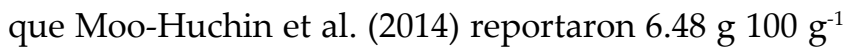
en base seca de fibra dietética soluble y $46.33{\mathrm{~g} 100 \mathrm{~g}^{-1}}^{-1}$ en base seca de fibra dietética total en frutos originarios de Yucatán, México.

En cuanto al contenido de pectinas, se han encontrado valores de $746 \mathrm{mg} 100 \mathrm{~g}^{-1}$ en la pulpa de nanche proveniente de El Cerrado, Brasil, mientras que, en muestras analizadas en México, los valores

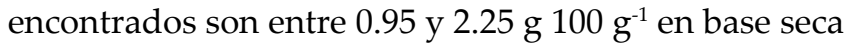
(Morzelle et al. 2015; Raya-Pérez et al. 2010). Además, se ha reportado que, durante el desarrollo del fruto, el contenido de pectina soluble aumenta de 0.03 a 0.13 por ciento de ácido galacturónico, y la característica principal de este proceso es el ablandamiento o senescencia del fruto (Da Silva et al. 2016). De igual manera, las semillas de nanche son una gran fuente de fibra dietética ( $27.5 \mathrm{~g}$ ) y contienen 12 por ciento de almidón (Araújo et al. 2018), mientras que la pulpa presenta 8.9 por ciento (De Souza et al. 2012).

Diversos autores han sugerido que las variaciones en las variables nutricionales y fisicoquímicas observadas en los frutos de nanche están asociadas 
a las condiciones ambientales de los lugares donde se encuentra la especie, así como a las características genéticas de las matrices (Morzelle et al. 2015).

\section{Componentes funcionales del fruto de nanche}

Los compuestos bioactivos se definen como los componentes de los alimentos que influyen en las actividades celulares y fisiológicas, cuya ingesta otorga un efecto benéfico para la salud; estos compuestos bioactivos no son nutrientes y, por tanto, no son esenciales para la vida. Normalmente, se encuentran en cantidades pequeñas en los alimentos que consumimos como parte de nuestra dieta habitual y en casi todos los casos provienen de fuentes alimentarias vegetales (Patil et al. 2009; Jiménez-Colmenero et al. 2013). Entre los compuestos bioactivos presentes en nanche se han encontrado, principalmente, fenólicos tales como: catequinas, taninos, así como también algunos carotenoides (Pires et al. 2019; Rodríguez et al. 2016; Mariutti et al. 2013; Jiménez et al. 2012). La investigación sugiere que el aumento del consumo de alimentos ricos en compuestos antioxidantes, como los compuestos fenólicos, se asocian a una reducción del riesgo de sufrir enfermedades crónicas (Almeida et al. 2011; Canuto et al. 2010).

Los compuestos fenólicos presentes en el fruto de nanche (Cuadro 3) superan lo reportado en piña, ciruela, papaya y guanábana (38.1, 55.0, 53.2, $54.8 \mathrm{mg}$ eag $100 \mathrm{~g}-1$ ), respectivamente (Almeida et al. 2011). En Panamá, se evaluaron los compuestos fenólicos de la pulpa de nanche, para obtener valores de ácido gálico que oscilaron entre 126.2 y 252.3 mg 100 g-1 y un porcentaje de inhibición entre 70 y 90 por ciento (Quintero et al. 2014). Asimismo, en México se analizaron extractos obtenidos de la pulpa de nanche, y se identificaron 23 compuestos de valor fitoquímico, con respecto a compuestos fenólicos totales fue de 291 mg eag 100 g-1 (López et al. 2014). Por otro lado, las semillas de nanche también presentan contenido de compuestos fenólicos (404 mg EAG100 g-1) y capacidad antioxidante (IC50=83\%) (Araújo et al. 2018). El grupo más importante de compuestos fenólicos en el nanche son los flavonoides, que consisten principalmente en antocianidinas, flavonoles, proantocianidinas (taninos condensados) y sus glicósidos (Rufino et al. 2010). Malta et al. (2013) reportaron un contenido de flavonoides de $311.6 \mathrm{mg}$ catequina $100 \mathrm{~g}-1$ en pulpa de nanche de Brasil, mientras que Moo-Huchin et al. (2014) mostraron un valor menor para la selección analizada en México (127.27 mg catequina 100 g-1).

Los principales flavonoides que se han encontrado en extractos de nanche son catequina, quercetina y rutina (López et al. 2014; Siguemoto 2013). La concentración de catequinas totales en extractos de pulpa de nanche originario de México mostraron un valor de $146 \mathrm{mg}$ catequina $100 \mathrm{~g}^{-1}$; esto indicó que, de los fenoles totales (291 mg EAG $100 \mathrm{~g} \mathrm{~g}^{-1}$ ), alrededor de 50 por ciento fueron catequinas (López et al. 2014). Gordon et al. (2011) reportaron la identificación de 19 compuestos polifenólicos en el extracto de la pulpa de nanche mediante HPLC, como galotaninos, galoil ácido quínico, proantocianidinas, derivados de quercetina y ácido gálico; también se han encontrado ácido ferúlico, resveratrol, catequinas, ácido cafeico, ácido ferúlico y kaempferol (Malta et al. 2013; Gordon et al. 2011). De la misma manera, 7 compuestos han sido identificados, basados en comparaciones con estándares comerciales: ácido gálico, catequina, (-) epicatequina, rutina, taxifolina y quercetina (Mariutti et al. 2013). Catequina y (-) epicatequina han sido identificados en corteza del árbol de nanche (Maldini et al. 2009); rutina y quercetina en hojas de nanche (Herrera et al. 2014). El principal compuesto fenólico encontrado tanto en pulpa como en extracto hidrofílico de nanche es quercetina (Mariutti et al. 2013). Para el caso del contenido de antocianinas, en estudios realizados por Sales y Waughon (2013) no se detectó este compuesto, situación contraria a lo reportado por Rufino et al. (2010), quienes encontraron $0.5 \mathrm{mg} 100 \mathrm{~g} \mathrm{~g}^{-1}$ de antocianina en pulpa fresca; otros autores reportan valores entre 1.02 y 3.29 por ciento (Almeida et al. 2011; Dos Santos et al. 2018; Moo-Huchin et al. 2014). En las semillas de nanche, es posible afirmar que sólo se encontraron trazas de estos compuestos (Araújo et al. 2018).

El contenido de carotenos reportados por Rufino et al. (2010) y De Souza et al. (2012) es de 1.1 y 1.25 mg $100 \mathrm{~g}^{-1}$ de fruto fresco, respectivamente. En pulpa de nanche se reporta una cantidad de carotenoides de 14.9 a $29.4 \mathrm{mg} 100 \mathrm{~g}^{-1}$, con predominancia de luteína; los carotenoides precursores de la síntesis de vitamina A ( $\beta$ - Criptoxantina y $\beta$-caroteno) se presentan en cantidades inferiores en relación con la luteína 
Cuadro 3. Compuestos bioactivos (fenoles totales, flavonoides, carotenoides y antocianinas) en frutos de nanche de diferentes regiones.

\begin{tabular}{|c|c|c|c|c|c|}
\hline Origen & $\begin{array}{c}\text { Fenoles totales } \\
\left(\mathrm{mg} \text { EAG } 100 \mathrm{~g}^{-1}\right)\end{array}$ & $\begin{array}{c}\text { Flavonoides } \\
\left(\mathrm{mg}^{-1} \text { EAG } 100 \mathrm{~g}^{-1}\right)\end{array}$ & 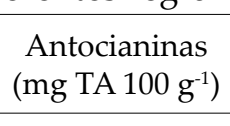 & $\begin{array}{c}\text { Carotenoides } \\
\text { (mg de } \beta \text {-caroteno } \\
\left.100 \mathrm{~g}^{-1}\right)\end{array}$ & Referencia \\
\hline \multicolumn{6}{|l|}{ Brasil } \\
\hline Alagoas & - & 6.77 & 3.29 & 1.64 & $\begin{array}{c}\text { Dos Santos et al. } \\
\text { (2018) }\end{array}$ \\
\hline Itapirapua & 222 & 311.6 & - & - & Malta et al. (2013) \\
\hline Minas Gerais & 334.4 & - & - & 1.25 & De Souza et al. (2012) \\
\hline Fortaleza & 159.9 & - & 1.02 & - & Almeida et al. (2011) \\
\hline Fortaleza & 238.0 & 13.8 & 0.5 & 1.1 & Rufino et al. (2010) \\
\hline \multicolumn{6}{|l|}{ México } \\
\hline Yucatán & 240.8 & 127.27 & 0.37 & 14.54 & $\begin{array}{l}\text { Moo-Huchin et al. } \\
\text { (2014) }\end{array}$ \\
\hline
\end{tabular}

(Siguemoto 2013). Rodríguez et al. (2016) identificaron 35 ésteres de carotenoides en pulpa de nanche, entre los cuales, luteína, $\beta$-caroteno y violaxantina son los más relevantes. En el análisis realizado por Irías-Mata et al. (2018), en nanche comercial (amarillo y rojo), identificaron a (all-E)-luteina, (all-E)-zeaxantina y (allE)-caroteno como los carotenoides predominantes; la (all-E)-luteína contribuyó a 80-89 por ciento del total de carotenoides presentes. En general, se encontraron concentraciones mayores de carotenoides totales en la

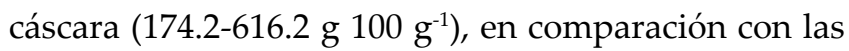

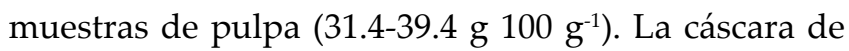
los frutos amarillos contenía concentraciones de carotenoides significativamente más altas (616.2 g 100

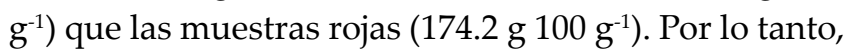
el nanche puede acumular carotenoides $y$, en algunas variedades, también antocianinas como indicadores de la madurez, y atraer fuentes dispersoras de semillas (Irías-Mata et al. 2018). Extractos de pulpa de nanche obtenidos por extracción supercrítica con $\mathrm{CO}_{2}$ mostraron altas cantidades de luteína $\left(209.73 \mu \mathrm{g} \mathrm{g}^{-1}\right)$, lo que confirma que el tipo de extracción, combinado con un solvente como el etanol, proporciona mayor selectividad, pureza y rendimiento (Pires et al. 2019). Asimismo, se ha reportado la concentración de vitamina E presente en el nanche, el cual fue representado por $\alpha$-tocoferol y $\beta$-tocotrienol, correspondiente a $52.3 \mathrm{y}$ 47.7 por ciento del contenido total, respectivamente (Hamacek et al. 2014).
Los carotenoides y compuestos fenólicos han reportado una alta correlación con la actividad antioxidante (Malta et al. 2013; Almeida et al. 2011; Rufino et al. 2010). Existen alimentos que presentan uno o varios antioxidantes con diferentes mecanismos de acción; por ello, la actividad antioxidante debe evaluarse con distintos métodos que puedan direccionarse a los diferentes mecanismos (Ramful et al. 2011; Moo-Huchin et al. 2014). Los métodos ampliamente usados son ABTS y DPPH (Almeida et al. 2011). El método ABTs está generalmente indicado para evaluar la actividad antioxidante de los compuestos hidrófilos, y el método dpph se usa comúnmente para extractos acuosos/orgánicos con compuestos hidrófilos y lipófilos (Rufino et al. 2010). En el Cuadro 4 se muestran las capacidades antioxidantes medidas por diferentes métodos en pulpa de nanche de Brasil y México.

Es importante tener en cuenta que el contenido de antioxidantes en frutos frescos puede verse afectado por la madurez, las prácticas agrícolas, la temperatura y las condiciones de almacenamiento, así como los tipos de estrés a los que están expuestas los frutos, como el almacenamiento a alta temperatura, la radiación ultravioleta, entre otros; por lo tanto, la presencia de altas cantidades de compuestos bioactivos en tejidos frescos no asegura su biodisponibilidad una vez que se consumen (González-Aguilar et al. 2008). 
Cuadro 4. Capacidad antioxidante en frutos de nanche de diferentes regiones.

\begin{tabular}{lcccc}
\hline \multicolumn{1}{c}{ Origen } & $\begin{array}{c}\text { ABTS } \\
(\mathrm{mmol} \text { Trolox/g) }\end{array}$ & $\begin{array}{c}\text { DPPH } \\
(\mathrm{mmol} \text { Trolox/g) }\end{array}$ & $\begin{array}{c}\text { FRAP } \\
(\mathrm{mmol} \text { Trolox/g) }\end{array}$ & Referencia \\
\hline Brasil & & & & \\
\cline { 1 - 1 } Minas & 57.3 & - & - & De Souza et al. (2012) \\
Gerais & 15.7 & 6.5 & - & Almeida et al. (2011) \\
Fortaleza & 412 & 238 & & Rufino et al. (2010) \\
Brasil & & & 334 & Moo-Huchin et al. (2014) \\
México & 661.8 & 372.9 & &
\end{tabular}

\section{Aplicación de compuestos funcionales de nanche}

En los últimos años, la relación entre el consumo de frutas y la salud humana ha sido el punto focal de la investigación científica. Este interés se debe a la búsqueda específica de los compuestos contenidos en vegetales que proporcionan beneficios a la salud del consumidor (Palafox-Carlos et al. 2011).

Por ello, esta relación entre el consumo de frutas y la salud humana ha tenido un cambio importante en los últimos años, debido a la continua aparición de evidencias científicas que acreditan cómo a través del consumo de sus componentes se modulan funciones fisiológicas en el organismo, lo cual favorece el bienestar y la salud (Jiménez-Colmenero 2013). En la actualidad, los problemas de salud pública se centran principalmente en el envejecimiento de la población y en el aumento de la prevalencia de determinadas enfermedades crónicas (Herrera et al. 2014). En tal sentido, se producen continuamente avances en el desarrollo de alimentos percibidos más saludables, fundamentados en la presencia de compuestos biológicamente activos (Yahia 2010). Últimamente, los fitoquímicos con gran potencial antioxidante han despertado el interés en las tecnologías de alimentos, ya que proporcionan protección contra la degradación oxidativa de los radicales libres (Jiménez et al. 2011). Además de sus propiedades antioxidantes, poseen una amplia gama de efectos biológicos, como antibacterianos, antivirales, antiinflamatorios, antitrombóticos y acciones vasodilatadoras (Di Carlo et al. 1999).

Por su parte, los compuestos extraídos de diferentes tejidos de B. crassifolia han mostrado propiedades como: antimicrobial (Carlos et al. 2017), además de presentar un efecto antiinflamatorio (Maldini et al. 2009), antidepresivo (Herrera et al. 2014) y como agente antidiabético (Pérez-Gutiérrez et al. 2010). La actividad antimicrobiana de los extractos etéreos y etanólicos de la corteza, pulpa y semilla de nanche ha sido probada para inhibición de bacterias Gram-positivas y Gram-negativas, donde ambos presentaron considerable potencial antimicrobiano, aunque el extracto de la semilla no presentó inhibición (Carlos et al. 2017). Sin embargo, a pesar de que el extracto de la cáscara ha sido eficiente en la inhibición de las bacterias Gram-negativas (Escherichia coli), no impide el crecimiento de Listeria monocytogenes. En general, los extractos de nanche presentaron un mayor potencial antimicrobiano contra bacterias Gram-positivas ( $S$. aureus) (Carlos et al. 2017). Esto puede ser explicado por la composición de las membranas celulares de las bacterias: las Gram-negativas presentan una membrana celular más compleja, debido a la presencia de lipopolisacáridos; por lo tanto, generalmente son menos susceptibles a la acción de agentes inhibidores de crecimiento, es decir, son más resistentes a la acción de agentes antimicrobianos (Pinho et al. 2012). Por otra parte, el extracto hidrofílico de fruto de nanche ha demostrado ser capaz de eliminar todas las especies reactivas de oxígeno (ROS) y nitrógeno reactivo (RNS), y es un fuerte eliminador de ácido hipocloroso (HOCL); asimismo, es capaz de inhibir la peroxidación lipídica en $48.0 \pm 5.0$ por ciento a la concentración más alta probada $\left(1000 \mu \mathrm{g} \mathrm{mL}^{-1}\right)$; además, ha sido capaz de proteger a las proteínas, lo que se indica mediante la inhibición de la oxidación de la hemoglobina $\left(\mathrm{IC}_{50}=\right.$ $271.0 \pm 44.0 \mu \mathrm{g} \mathrm{mL}^{-1}$ ), y puede inhibir el daño oxidativo inducido por peroxilo (ROO) en eritrocitos humanos, 
como lo demuestran diferentes biomarcadores de estrés oxidativo (Mariutti et al. 2014). Por lo tanto, el nanche puede considerarse una fuente prometedora de compuestos bioactivos para ser utilizados por las industrias farmacéutica y alimentaria, pues la inhibición de las acciones oxidativas de Ros y RNS por compuestos antioxidantes es una propiedad crucial, gracias a la cual se puede mitigar el desarrollo de estrés oxidativo relacionado a enfermedades (Mariutti et al. 2014).

Algunos estudios sugieren que la aplicación tópica de antioxidantes puede prevenir o retrasar el daño cutáneo inducido por radiación ultravioleta (UV-B), al proporcionar un efecto fotoquimoprotector y fortalecer el sistema de protección endógeno, lo que, en última instancia, reduciría el daño oxidativo en la piel (Salucci et al. 2014). En este contexto, se ha prestado mucha atención a los productos naturales que poseen propiedades antioxidantes y antiinflamatorias. Aunque la piel tiene varios mecanismos de defensa para evitar el daño oxidante, la exposición prolongada a la radiación ultravioleta (Uv) puede aumentar la formación de Ros y causar un desequilibrio oxidante (Terra et al. 2012). Las Ros pueden reaccionar con moléculas orgánicas (proteínas, carbohidratos, ácidos nucleicos y, especialmente, lípidos) y causar pérdida de integridad celular, peroxidación de lípidos y la liberación de citoquinas. Este proceso finalmente causará lesiones en la piel y acelerará el envejecimiento, así como el desarrollo de enfermedades malignas (Peres et al. 2011). De Souza et al. (2017) llevaron a cabo un estudio con piel de oreja de cerdo y se demostró que el extracto de hojas de nanche tiene una excelente penetración de compuestos fenólicos en la epidermis/dermis, lo que proporcionó un aumento en el potencial antioxidante de la piel. Gracias a estos resultados se supo que la fracción obtenida de las hojas puede ser una buena fuente de catequinas y otros compuestos fenólicos, los cuales podrían ejercer un efecto fotoquimoprotector cuando se incorpore en formulaciones tópicas.

El nanche también ha demostrado poseer propiedades antihiperglucémicas significativas; después de $4 \mathrm{~h}$ de una dosis oral única, puede mejorar la hiperlipidemia e hiperinsulinemia en ratas diabéticas inducidas por estreptozotocina. Asimismo, extractos de pulpa y semilla han mostrado una actividad inhibitoria significativa contra la formación de AGE (productos finales de glicación avanzada), con valores IC (50) que oscilan entre 94.3 y $138.7 \mu \mathrm{g} \mathrm{mL-1;} \mathrm{por} \mathrm{lo}$ tanto, el nanche puede considerarse como un posible agente anti-diabético seguro (Pérez-Gutiérrez et al. 2010). De igual manera, Matildes et al. (2017) evaluaron productos de panificación elaborados con harina de pulpa y semilla de nanche, donde se presentaron porcentajes de inhibición de formación de AGE bastante cercanos entre sí, antes y después del proceso de horneado, lo cual demuestra que los compuestos bioactivos presentes en el nanche no fueron afectados por la temperatura y el tiempo de horneado.

Las Ros provocan una oxidación que da lugar a la desintegración de la membrana celular, así como daño a la proteína de la membrana, seguido de la mutación del ADN, lo que provoca el desarrollo de diversas enfermedades como el cáncer (Xiao et al. 2014). Extractos obtenidos de fruto de nanche se adicionaron a células in vitro de cáncer de hígado (HepG2) para determinar si podrían inhibir la proliferación de células tumorales; las células HepG2 fueron tratadas con extractos equivalentes a 25.0 y $95.0 \mathrm{mg} \mathrm{mL}^{-1}$ de nanche, y los resultados mostraron una actividad antiproliferativa de $173.6 \pm 18.2 \mathrm{mg} \mathrm{mL}^{-1} \mathrm{y}$ antioxidante celular (41.36 \pm $17.89 \mathrm{mmol}$ quercetina $100 \mathrm{~g}^{-1}$ en pulpa de nanche); esto indica que, los extractos analizados tienen potentes efectos inhibidores sobre el crecimiento de las células HepG2 y la inhibición de la proliferación celular se observó de forma dependiente de la dosis después de la exposición a los extractos (Malta et al. 2013).

Por otra parte, Herrera et al. (2014) evaluaron los efectos ansiolíticos, anticonvulsivos, antidepresivos y sedantes producidos por los extractos de B. crassifolia y su influencia sobre la actividad motora en ratones, luego de lo cual demostraron que el extracto metanólico produce un efecto antidepresivo en la prueba de natación forzada en ratones a dosis de $500 \mathrm{mg} \mathrm{kg}^{-1}$; también se confirmó que no posee propiedades ansiolíticas, sedantes o anticonvulsivas, y no causa reducción de locomoción de ratones. La hesperidina y la quercetina son los flavonoides a los que se les considera como los responsables de dichos efectos (Herrera et al. 2014); además, se ha relacionado a los ácidos grasos insaturados que se encuentran presentes en el nanche, con efectos terapéuticos en el tratamiento y prevención de enfermedades cardiovasculares, como aterosclerosis, trombosis e hipercolesterolemia, ya que 
este vínculo se observó para otros alimentos (Pires et al. 2019).

\section{Conclusiones}

En síntesis, el nanche tiene una gran variedad de efectos biológicos que se muestran en diversos estudios in vivo e in vitro; sin embargo, ha sido subestimado en el terreno de la prevención de enfermedades. Se requieren estudios adicionales para aclarar los mecanismos detallados de estas diversas acciones, incluidos los efectos sinérgicos de los componentes funcionales. La presente revisión incita a reconocer el valor nutricional del nanche, el cual puede contribuir a suministrar necesidades nutricionales diarias, particularmente en fibra dietética y vitamina $\mathrm{C}$, así como también en compuestos antioxidantes, lo que justifica el fomento de su consumo y uso para el desarrollo de alimentos funcionales. 


\section{Literatura Citada}

Abadio FDB, Silva IG, De Oliveira RB. 2012. Physicochemical characteristics and antioxidant activity of three native fruits from Brazilian savannah (Cerrado). Alimentos e Nutrição (Araraquara) 23: 179-185.

Almeida MMB, De Sousa PHM, Arriaga ÂMC, do Prado GM, Magalhães CEdeC, Maia GA, De Lemos TLG. 2011. Bioactive compounds and antioxidant activity of fresh exotic fruits from northeastern Brazil. Food Research International 44: 2155-2159. http://doi.org/10.1016/j.foodres.2011.03.051

Araújo ACMA, Menezes EGT, Terra AWC, Dias BO, De Oliveira ER, Queiroz F. 2018. Bioactive compounds and chemical composition of Brazilian Cerrado fruits' wastes: Pequi almonds, murici, and sweet passionfruit seeds. Food Science and Technology 38 (Supl.1): 203-214. http://doi.org/10.1590/fst.19417

Argueta CZA, Mata S. 2009. Atlas de las plantas de la medicina tradicional mexicana. Byrsonima crassifolia (L.) Kunth. [citado 2019 septiembre 20]. Disponible en: http://www.medicinatradicionalmexicana.unam.mx/ monografia.php?1=3\&t=Nanche\&i $d=7985$.

Caballero A, Vela G, Pérez J, Escobar R, Ballinas J. 2012. Uso del nanche (Byrsonima crassifolia (L.) Kunth) en gelatina artesanal para niños. Etnobiología 10: 50-55.

Canuto GAB, Xavier AAO, Neves LC, Benassi MDT. 2010. Caracterização físico-química de polpas de frutos da Amazônia e sua correlação com a atividade antiradical livre. Revista Brasileira de Fruticultura 32: 11961205. http://doi.org/10.1590/S0100-29452010005000122

Carlos NDA, Loss RA, e Silva SS, Guedes FS, Carvalho JWP. 2017. Avaliação físico-química e atividade antimicrobiana da casca, polpa e semente do murici (Byrsonima crassifolia). Enciclopedia Biosfera 14: 232243. http://doi.org/10.18677/EnciBio_2017A22

[CONABIO] Comisión Nacional para el Conocimiento y Uso de la Biodiversidad. [internet]. 2019. Catálogo de autoridades taxonómicas de especies de flora y fauna con distribución en México. Base de datos SNIB-CONABIO. [citado 2019 septiembre 20]. Disponible en: http://www.snib.mx/descargasSNIBmx/SNIBTaxonomia_20200322_171728.zip.

Costa EA, Almeida BS, Machado PH, Camurça APS, Barbosa EC, Lima LGN. 2017. Estructurado de murici (Byrsonima crassifolia (L.) Kunth) a base de hidro- coloide: elaboração e caracterização físico-química. Arquivos Brasileiros de Alimentaçao, Recife 2 (3): 174-181.

Da Silva PMC, Neves LC, Bastos VJ, Lima CGB, De Araújo KG, Roberto SR. 2016. Harvesting period of Murici (Byrsonima crassifolia Kunth) fruit in relation to physical and chemical parameters evaluated during fruit development. Scientia Horticulturae 200: 66-72. http://doi.org/10.1016/j.scienta.2015.12.041

De Souza VR, Pereira PAP, Queiroz F, Borges SV, Carneiro JDS. 2012. Determination of bioactive compounds, antioxidant activity and chemical composition of Cerrado Brazilian fruits. Food Chemistry 134: 381386. http://doi.org/10.1016/j.foodchem.2012.02.191

De Souza RO, Alves GdeAD, Forte ALSA, MarqueleOliveira F, Da Silva DF, Rogez H, Fonseca MJV. 2017. Byrsonima crassifolia extract and fraction prevent UVBinduced oxidative stress in keratinocytes culture and increase antioxidant activity on skin. Industrial Crops and Products 108: 485-494. http://doi.org/10.1016/j. indcrop.2017.07.015

Di Carlo G, Mascolo N, Izzo AA, Capasso F. 1999. Flavonoids: Old and new aspects of a class of natural therapeutic drugs. Life Sciences 65: 337-353. http:// doi.org/10.1016/s0024-3205(99)00120-4

Dos Santos JFL, Dos Santos DB, Rossi AAB, Pena GF, Tiago AV. 2018. Caracterização biométrica de frutos e sementes de murici (Byrsonima crassifólia L.) na região norte do Mato Grosso, Brasil. Enciclopédia Biosfera 15: 1-12. http://doi.org/10.18677/EnciBio_2018A24

González-Aguilar G, Robles-Sánchez RM, MartínezTéllez MA, Olivas GI, Álvarez-Parrilla E, De la Rosa LA. 2008. Bioactive compounds in fruits: Health benefits and effect of storage conditions. Stewart Postharvest Review 4: 1-10. http://doi.org/10.2212/ spr.2008.3.8

Gordon A, Jungfer E, Da Silva BA, Maia JGS, Marx F. 2011. Phenolic constituents and antioxidant capacity of four underutilized fruits from the Amazon region. Journal of Agricultural and Food Chemistry 59: 76887699. http://doi.org/10.1021/jf201039r

Hamacek FR, Martino HSD, Pinheiro-Sant'Ana HM. 2014. Murici, fruit from the Cerrado of Minas Gerais, Brazil: Physical and physicochemical characteristics, and occurrence and concentration of carotenoids and vitamins. Fruits 69: 459-472. https://doi.org/10.1051/ fruits/2014032 
Herrera F, Betancur D, Segura MR. 2014. Compuestos bioactivos de la dieta con potencial en la prevención de patologías relacionadas con sobrepeso y obesidad: péptidos biológicamente activos. Nutrición Hospitalaria 29: 10-20. https:/doi.org/10.3305/ nh.2014.29.1.6990

Herrera-Ruiz M, Zamilpa A, González-Cortazar M, Reyes-Chilpa R, León E, García MP, Huerta RM. 2011. Antidepressant effect and pharmacological evaluation of standardized extract of flavonoids from Byrsonima crassifolia. Phytomedicine 18: 1255-1261. https://doi.org/10.1016/J.PHYMED.2011.06.018

Irías-Mata A, Jiménez VM, Steingass CB, Schweiggert RM, Carle R, Esquivel P. 2018. Carotenoids and xanthophyll esters of yellow and red nance fruits (Byrsonima crassifolia (L.) Kunth) from Costa Rica. Food Research International 111: 708-714. https://doi. org/10.1016/j.foodres.2018.05.063

Jiménez-Colmenero F. 2013. Emulsiones múltiples; compuestos bioactivos y alimentos funcionales. Nutrición Hospitalaria 28: 1413-1421. https://doi.org/10.3305/ nh.2013.28.5.6673

Jiménez AM, Sierra CA, Rodríguez-Pulido FJ, GonzálezMiret ML, Heredia FJ, Osorio C. 2011. Physicochemical characterisation of gulupa (Passiflora edulis Sim. fo edulis) fruit from Colombia during the ripening. Food Research International 44: 1912-1918. https://doi.org/10.1016/j.foodres.2010.11.007

Jiménez HD, Solis FJA, Vargas N, Muñiz IE, Melo V, Schettino B. 2012. Estudio etnobotánico de Byrsonima crassifolia L (K) y su composición nutricional. Revista Latinoamericana de Química 39 (Supl. Esp.): 196.

López NEA, Manchón N, Herrera J. 2014. Componentes funcionales en Nanche (Byrsonima crassifolia (L) Kunth). En: Ramos M, Aguilera V, editores. Ciencias Agropecuarias, Handbook. Valle de Santiago, ECORFAN. P. 6-22.

Maldini M, Sosa S, Montoro P, Giangaspero A, Balick MJ, Pizza C, Della-Loggia R. 2009. Screening of the topical anti-inflammatory activity of the bark of Acacia cornigera Willdenow, Byrsonima crassifolia Kunth, Sweetia panamensis Yakovlev and the leaves of Sphagneticola trilobata Hitchcock. Journal of Ethnopharmacology 122: 430-433. https://doi.org/10.1016/j.jep.2009.02.002

Malta LG, Tessaro EP, Eberlin M, Pastore GM, Hai RH. 2013. Assessment of antioxidant and antiproliferative activities and the identification of phenolic of exotic
Brazilian fruits. Food Ressearch International 53: 417425. https://doi.org/10.1016/j.foodres.2013.04.024

Mariutti LRB, Rodrigues E, Mercadante AZ. 2013. Carotenoids from Byrsonima crassifolia: Identification, quantification and in vitro scavenging capacity against peroxyl radicals. Journal of Food Composition and Analysis 31: 155-160. https://doi.org/10.1016/j. jfca.2013.05.005

Mariutti LRB, Rodrigues E, Chisté RC, Fernandes E, Mercadante AZ. 2014. The Amazonian fruit Byrsonima crassifolia effectively scavenges reactive oxygen and nitrogen species and protects human erythrocytes against oxidative damage. Food Research International 64: 618-625. http://doi.org/10.1016/j.foodres.2014.07.032

Matildes A, Pérez RM, Gallardo YT. 2017. Elaboración de productos de panificación utilizando nanche como ingrediente con componentes funcionales. Revista Electrónica Humanidades, Tecnología y Ciencia 9: 1-6.

Medina-Torres R, Juárez-López P, Salazar-García S, López-Guzmán G, Ibarra-Sánchez L, Arrieta-Ramos BG, Martínez-Moreno E. 2015. Evaluación de calidad en frutos de 41 genotipos de nanche (Byrsonima crassifolia L. HBK) de Nayarit, México. Revista Mexicana de Ciencias Agrícolas 6: 253-264. https://doi.org/10.29312/ remexca.v6i2.686

Monteiro DCB, De Sousa WC, Pires CRF, Azevedo LA, Borges JS. 2015. Caracterização físico-química do fruto e da geleia de murici (Brysonima crassifolia). Enciclopédia Biosfera 11: 3356-3366.

Moo-Huchin VM, Estrada-Mota I, Estrada-León R, CuevasGlory L, Ortiz-Vázquez E, Vargas ML, BetancurtAncona D, Sauri-Duch E. 2014. Determination of some physicochemical characteristics, bioactive compounds and antioxidant activity of tropical fruits from Yucatan, Mexico. Food Chemistry 151: 508-515. https:// doi.org/10.1016/j.foodchem.2013.12.013

Moreno-Martínez JL, Ruiz-Bello C, Jasso-García Y, Moreno-Ruiz M, Villarreal-Fuentes JM. 2016. Valor agregado del nanche (Byrsonima crassifolia (L.) H.B.K.). Agroproductividad 9: 66-71.

Morzelle MC, Bachiega PS, Souza EC, Vilas BC, Lamounier ML. 2015. Caracterização química e física de frutos de curriola, gabiroba e murici provenientes do Cerrado brasileiro. Revista Brasileira de Fruticultura 37: 96-103. https://doi.org/10.1590/0100-2945-036/14

Palafox-Carlos H, Ayala-Zavala JF, González-Aguilar GA. 2011. The role of dietary fiber in the bioaccessi- 
bility and bioavailability of fruit and vegetable antioxidants. Journal of Food Science 76: 6-15. https://doi. org/10.1111/j.1750-3841.2010.01957.x

Patil BS, Jayaprakasha GK, Chidambara KN, Vikram A. 2009. Bioactive compounds: Historical perspectives, opportunities, and challenges. Journal of Agricultural and Food Chemistry 57: 8142-8160. https://doi.org/10.1021/ jf9000132

Pereira AC, Dos Santos ER. 2015. Frutas nativas do Tocantins com potencial de aproveitamento econômico. AgriEnviromental Sciences 1: 22-37.

Pérez-Gutiérrez RM, Muñiz-Ramírez A, Gómez Y, Bautista E. 2010. Antihyperglycemic, antihyperlipidemic and antiglycation effects of Byrsonima crassifolia fruit and seed in normal and streptozotocin-induced diabetic rats. Plant Foods for Human Nutrition 65: 350-357. https:// doi.org/10.1007/s11130-010-0181-5

Peres PS, Terra VA, Guarnier FA, Cecchini R, Cecchini AL. 2011. Photoaging and chronological aging profile: Understanding oxidation of the skin. Journal of Photochemistry and Photobiology B: Biology 103: 93-97. https://doi.org/10.1016/j.jphotobiol.2011.01.019

Pimentel R, Guimarães FN, Santos VM, Resende JCF. 2010. Qualidade pós-colheita dos genotipos de banana PA4244 e Prata-Anã cultivados no norte de Minas Gerais. Revista Brasileira de Fruticultura 32: 407-413. https://doi. org/10.1590/S0100-29452010005000047

Pinho L, Souza PNS, Sobrinho EM, Almeida AC, Martins ER. 2012. Atividade antimicrobiana de extratos hidroalcoolicos das folhas de alecrim- pimenta, aroeira, barbatimão, erva baleeira e do farelo da casca de pequi. Ciência Rural 42: 326-331. https://doi.org/10.1590/ S0103-84782012005000003

Pires VP, Borges S, Santana AA, Sampaio RM, Villa-Vélez H. 2017. Estudio fisicoquímico e reológico da pulpa de murici. XII Congreso Brasileiro de Engenharia Quimica em Iniciacao Cientifica. Sao Carlos, Sao Paulo, Brasil. 1: 934939. https://doi.org/10.5151/chemeng-cobeqic2017-161

Pires FCS, Silva APS, Salazar MAR, Da Costa WA, Da Costa HSC, Lopes AS, De CarvalhoJRN. 2019. Determination of process parameters and bioactive properties of the murici pulp (Byrsonima crassifolia) extracts obtained by supercritical extraction. The Journal of Supercritical Fluids 146: 128-135. https://doi.org/10.1016/j.supflu.2019.01.014

Quintero R, González R, Soo A, McLean R, Sevillano A. 2014. Estudio preliminar de las propiedades antioxidantes y compuestos fenólicos en extractos de frutos de nance (Byrsonima Crassifolia). 3era International Conference on Food Innovation-FoodInnova. Concordia, Provincia Entre Ríos, Argentina.

Ramful D, Tarnus E, Aruoma OI, Bourdon E, Bahorun T. 2011. Polyphenol composition, vitamin $C$ content and antioxidant capacity of Mauritian citrus fruit pulps. Food Research International 44: 2088-2099. https://doi. org/10.1016/j.foodres.2011.03.056

Raya-Pérez JC, Aguirre-Mancilla CL, Gil-Vega K, Simpson J. 2010. La domesticación de plantas en México: comparación de la forma cultivada y silvestre de Byrsonima crassifolia (Malpighiaceae). Polibotánica 30: 239-256.

Rivas-Castro SF, Martínez-Moreno E, Alia-Tejacal I, Pérez-López A. 2019. Physical and physiological changes in phenotypes of nance [Byrsonima crassifolia (L.) H.B.K.] with different harvest maturity. Scientia Horticulturae 256: 108620. https://doi.org/10.1016/j. scienta.2019.108620

Rodríguez SM, Moura EF, Ramos GKS, Oliveira MSP. 2016. Genetic variability analysis of Byrsonima crassifolia germplasm collected in Pará state using ISSR markers. Genetics and Molecular Research 15 (4). https://doi. org/10.4238/gmr15048887

Rufino MSM, Alves RE, De Brito ES, Pérez-Jiménez J, SauraCalixto F, Mancini-Filho J. 2010. Bioactive compounds and antioxidant capacities of 18 non-traditional tropical fruits from Brazil. Food Chemistry 121: 996-1002. https://doi.org/10.1016/J.FOODCHEM.2010.01.037

Sales A, Waughon TGM. 2013. Influence of processing on the bioactive compound content in murici and hog plum fruits. Revista Agrarian 6: 7-15.

Salucci S, Burattini S, Curzi D, Buontempo F, Martelli AM, Zappia G, Falcieri E, Battistelli M. 2014. Antioxidants in the prevention of UVB-induced keratynocyte apoptosis. Journal of Photochemistry and Photobiology B: Biology 141: 1-9. https://doi.org/10.1016/j.jphotobiol.2014.09.004 [SIAP] Servicio de Información Agroalimentaria y Pesquera. [internet]. 2018. Cierre de la producción Agrícola 2018. [citado 2019 septiembre 20]. Disponible en: https://nube.siap.gob.mx/cierreagricola/.

Siguemoto ÉS. 2013. Composição nutricional e propriedades funcionais do murici (Byrsomina crassifolia) e moringa (Moringa oleifera). Tesis de Maestría. Faculdade de Saúde Pública, São Paulo. São Paulo, Brasil. https://doi. org/10.11606/D.6.2013.tde-25092013-083726

Terra VA, Souza-Neto FP, Pereira RC, Silva TNX, Costa ACC, Luiz RC, Cecchini R, Cechini AL. 2012. Time- 
dependent reactive species formation and oxidative stress damage in the skin after UVB irradiation. Journal of Photochemistry and Photobiology B: Biology 109: 3441. https://doi.org/10.1016/j.jphotobiol.2012.01.003

[USDA] United States Department of Agriculture. [internet]. 2018. FoodData Central. [citado 2019 septiembre 20]. Disponible en: https://fdc.nal.usda.gov/fdc-app. html\#/food-details/167789/nutrients.

Xiao J, Muzashvili TS, Georgiev MI. 2014. Advances in the biotechnological glycosylation of valuable flavonoids. Biotechnology Advances 32: 1145-1156. https://doi.org/10.1016/j.biotechadv.2014.04.006

Yahia EM. 2010. The contribution of fruit and vegetable consumption to human health. En: De la Rosa LA, AlvarezPadilla E, González GA, editores. Phytochemicals: Chemistry, Nutritional and Stability. Hoboken, WileyBlackwell. P. 3-51. 